\title{
Defect Detection Based on Segmentation of Thermographic Images of Frequency Modulated Thermal Wave Imaging
}

\author{
V.Phani Bhushan, K. Murali, K.S. Sagar Reddy
}

\begin{abstract}
To improve the usefulness of the data, the raw images acquired during non-destructive testing should be processed by image processing techniques. In this paper, by Frequency Modulated Thermal Wave Imaging, we use the image fusion technique to boost the detection capability of defects in a GFRP sample with 25 squared Teflon inserts of different sizes positioned at various depths. In applications such as detection, image segmentation is useful where it is difficult to process the entire image at a time. In this paper, Adaptive Thresholding Image segmentation is used to classify the delamination in Thermographic Images of Infrared Non-Destructive Research on images captured at two different times. Image fusion is later applied to segmented images. Image fusion is used to merge two or more pictures of a different focus and to provide the best picture quality. Fusion is carried out using the Basic Averaging Method here. Using Relative Foreground Area error, the performance of the proposed method is quantitatively assessed. The region and shape of an object are important parameters in the case of Non-Destructive Evaluation. Such parameters are contrasted with current methods of segmentation.

Keywords: - NDT, Adaptive Thresholding, Image Fusion, Quantitative Analysis.
\end{abstract}

\section{INTRODUCTION}

The basic concept of Non-Destructive Testing ${ }^{[1]}$ and Assessment is the energy pumped into the structure to determine the state of the substance (identification of delamination, void, etc.). It was categorized into the following categories depending on the type of excitation, such as ultrasonic imaging, radiographic or eddy current, heat energy (infrared testing), vibration, etc. Due to its quick inspection rate, non-contact, portability, and ease of interpretation, infrared thermography has become the most common non-destructive testing method to evaluate subsurface defects in metallic and composite materials. Although different methods for the detection of delamination defects in solid materials have been proposed in the past, three of them are mainly in use: pulse thermography (PT), lock thermography (LT), and pulse phase thermography (PPT). But every technique has its own constraints:

Manuscript received on February 13, 2021

Revised Manuscript received on February 25, 2021.

Manuscript published on February 28, 2021

* Correspondence Author

V.Phani Bhushan*, Department of E.C. E, P.B.R.V.I.T.S. Kavali, A.P., India. E-mail: phani.vpb@gmail.com

K. Murali, Department of ECE, Narayana Engineering College, Nellore, A.P., India. E-mail: murali.kante@gmail.com

K.S. Sagar Reddy, Department of ECE, Narayana Engineering College., Nellore, A.P., India. E-mail: siddu.sindhu12@gmail.com

(C) The Authors. Published by Blue Eyes Intelligence Engineering and Sciences Publication (BEIESP). This is an open access article under the CC BY-NC-ND license (http://creativecommons.org/licenses/by-nc-nd/4.0/)
Pulse thermography (PT) requires heat sources of high peak power and is vulnerable to variations in surface emissivity and non-uniform heating on the test sample surface, lock-in thermography (LT) struggles with low depth resolution and long processing time, and Pulse Phase Thermography (PPT) requires heat sources of high peak power to detect deeper subsurface defects that may affect the test surface ${ }^{[2,3]}$.In this paper, by means of frequency modulated thermal wave imaging, glass fiber reinforced polymer (GFRP) samples with 25 square Teflon inserts of different sizes and located at different depths were investigated. The recorded thermal image from the thermal camera cannot provide exact information about the defects inside the sample. Thermal images captured are recorded at different time intervals and segmentation is done on the thermographic images at different time intervals. Segmentation helps to analyze thermograms quantitatively and qualitatively. Segmentation is helpful in finding the size, shape, and area of defects. Several segmentation methods are available viz., Thresholding, Clustering, and Edge detection. The unfocused areas in an image never deliver the best picture quality. Image fusion is then used to provide an image of the highest quality. In order to enhance defect detectability, image fusion techniques have been studied and addressed.

\section{EXPERIMENTAL SETUP}

An experiment is performed with 25 square-shaped Teflon inserts inserted into it on a glass fiber reinforced polymer sample. The Teflon inserts are positioned at various depths with different dimensions, as shown in Fig.1. To capture image sequences, an infrared camera [4] with a frame rate of $450 \mathrm{~Hz}$ and a temperature resolution of $0.001 \mathrm{~K}$ with a resolution of $320 \times 256$ pixels is used. The frequency modulated thermal wave stimulation with a frequency range from $0.01 \mathrm{~Hz}$ to $0.1 \mathrm{~Hz}$ in 100 seconds is applied to the sample using two $1 \mathrm{Kw}$ power halogen lamps. At a frame rate of $20 \mathrm{~Hz}$, the thermal response is recorded. The experimental configuration is shown in Fig.2.

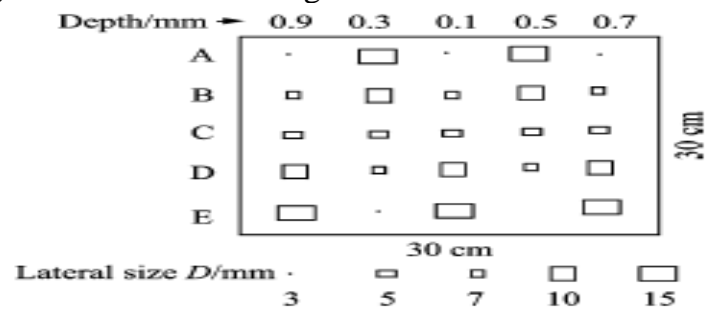

Fig.1 Layout of GFRP sample

Published By:

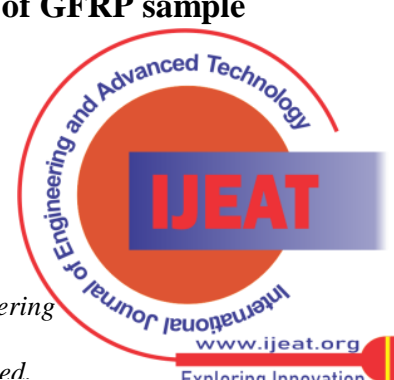

Blue Eyes Intelligence Engineering and Sciences Publication 


\section{Wave Imaging}

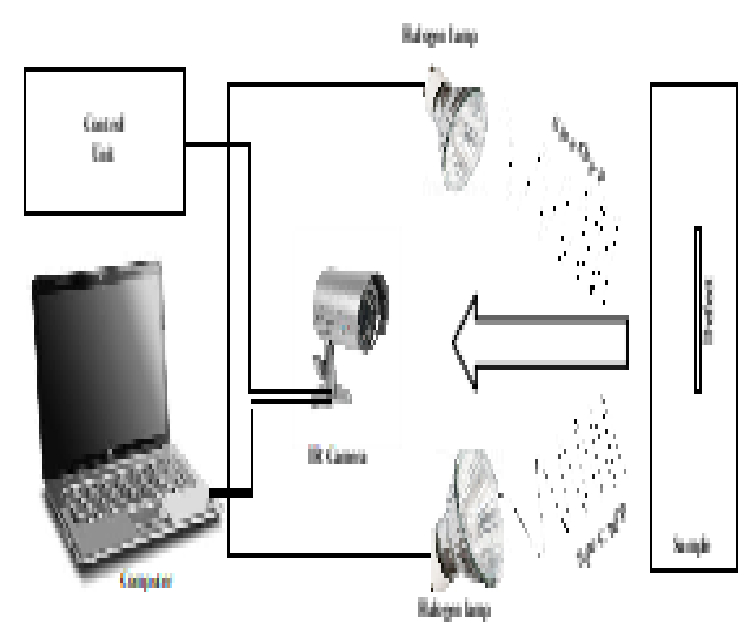

Fig.2 Experimental setup

\section{SEGMENTATION OF THERMAL IMAGES}

Thermal imaging is a way of using infrared radiation and thermal energy to obtain information about objects, often in low visibility conditions, to formulate images of them. In thermographic images, the identification of faulty areas is a major challenge. Image segmentation is an efficient tool for both quantitatively and qualitatively analyzing thermographic images. Segmentation is used in this paper to find the size and depth of defects for a GFRP material in thermographic images taken. Segmentation is achieved by demarcating an object from an image by using the object's pixel-level or object-level properties. Edges, texture, pixel intensity variation within the object, shape, size, and orientation ${ }^{[5]}$ can be these properties. There are two purposes to the segmentation. For further research, the first is to decompose an image into regions, and the second is to make a shift in the image representation for faster analysis. A single or a combination of segmentation techniques can be applied based on the application to effectively solve the problem [6] Essentially, there are three types of segmentation techniques, viz. Thresholding, Edge Detection, and Segmentations Based on Area. Thresholding [7] is one of the most widely used segmentation methods for the choice of a threshold value $\mathrm{T}$ to discriminate foreground from context. For NDT \& Assessment, this makes it appropriate. However, the efficiency of thresholding methods has the following limitations: small object size, large object, and background amplitude variances, the large amount of noise, etc. Several segmentation-based algorithms have been proposed for this purpose. Method of Otsu [8], Thresholding based on histogram $[9,15]$, Thresholding based on clustering [10], Entropic thresholding, and Adaptive Thresholding.

\section{PROPOSED SEGMENTATION METHOD}

Thresholding is applied in image processing to produce binary images from grayscale images. Thresholding is a nonlinear process that transforms a gray-scale image to a binary image where pixels below or above the defined threshold value are allocated to the two levels. Compared to traditional thresholding methods, adaptive thresholding is found to be higher. Some sections remain under more shadow in an image and sometimes illuminations influence the picture as well. In the traditional thresholding process, the mean value is taken as a global or normal threshold value. In an image, if a darker component or pixel contains a value greater than the

threshold value, it will appear in the foreground of the image. Similarly, if the value is smaller than the value of the threshold, the pixel or component appears in the background [11]. The resulting image of adaptive thresholding is a binary image since it shows the differences between different threshold values. The white region describes values below the threshold, and the black region describes values above the threshold. The Raw image obtained from the Thermal Camera is subjected to Adaptive Segmentation to extract the delamination's like size, depth, and area in Thermographic Images.

The algorithm assumes that two types of pixels are in the thermal image to be segmented (e.g., foreground and background)

The ideal threshold separating these two groups is then determined such that their cumulative distribution (intra-class variance) is negligible. This results in a binary image where pixels are set as 0 (background) and 1 (foreground).

Application of Segmentation to input raw images is recorded at different time instants such as $\mathrm{t}_{1}, \mathrm{t}_{2}, \ldots, \mathrm{t}_{\mathrm{n}}$.

These different segmented images are selected based on image resolution and visibility of defects.

In this case, segmented images at $16^{\text {th }}, 17^{\text {th }}$, and $18^{\text {th }}$ seconds are considered because of the visibility of all the defects in the respective images.

The segmented images after Adaptive Thresholding are shown below:

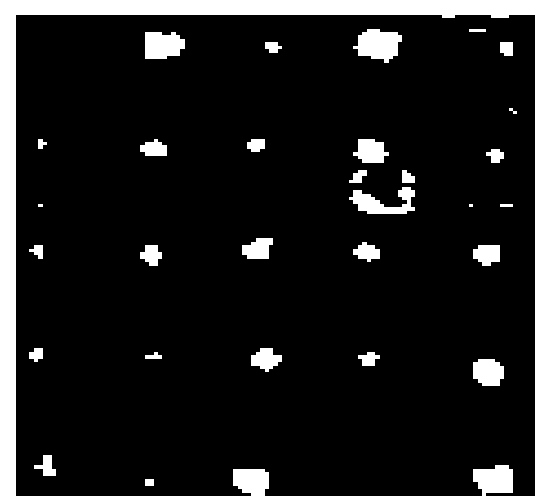

a.) Segmented Image at $16^{\text {th }} \mathrm{sec}$

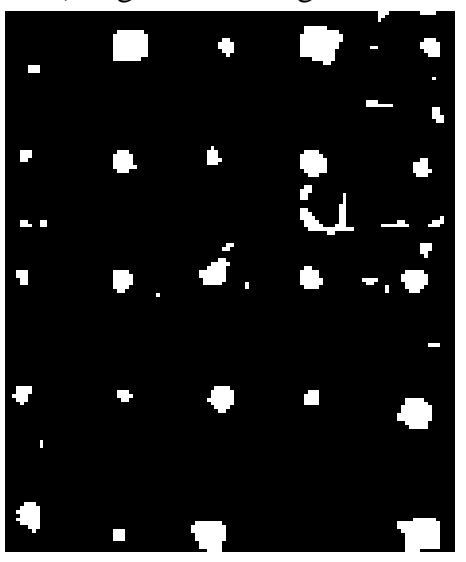

b.) Segmented Image at $17^{\text {th }} \mathrm{sec}$

Blue Eyes Intelligence Engineering and Sciences Publication

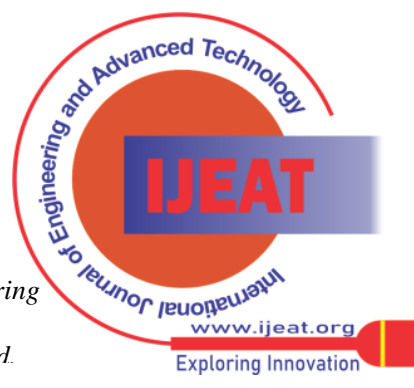




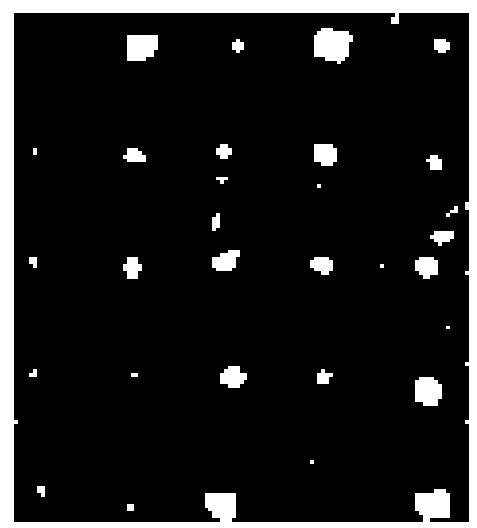

c.) Segmented Image at $18^{\text {th }} \mathrm{sec}$

Fig.3. a), b) and c) shows segmented images at $16^{\text {th }}, 17$ th and $18^{\text {th }}$ time instants after Adaptive Thresholding.

\section{IMAGE FUSION}

Picture fusion is the mechanism by which two images with different characteristics are combined. In an image, the unfocused regions never engage in the best quality of the images. The fusion is a method to combine the two or more images oriented differently and provide high quality for the completely focused image. Fusion applications have been playing a very important role in recent days. There is a great application of their concern for both the time domain and frequency domain approaches.

\section{A. Image Fusion Using Simple Averaging Method ${ }^{[12]}$ : -}

The average of the unfocused images is performed in this technique to get better-quality images. Let $\mathrm{x}$ and $\mathrm{y}$ be two images that have different areas of focus. The output fused image is given by $(x+y) / 2$ according to the fusion rule. Below is the resulting image after image fusion:

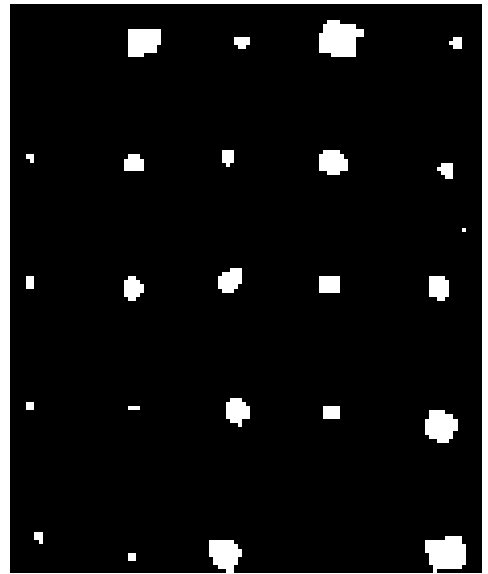

Fig.4. Resultant Image after Image Fusion

\section{PERFORMANCE EVALUATION}

Performance evaluation is an important criterion when proposing Thresholding Algorithm. The performance is evaluated quantitatively using Relative Foreground Area Error. In the case of NDE applications area and shape of the object are essential parameters for quantitative analysis. The value of Relative Foreground Area Error measures the quantity of segmentation. RAE is zero if it is a perfect match of segment regions. The penalty is maximum one if zero overlaps of object area occurs ${ }^{[13]}$.

$$
\begin{array}{rlr}
\operatorname{RAE} & =\left(A T-A_{M}\right) / A_{T} & A_{M}<A_{T} \\
& =\left(A_{M}-A_{T}\right) / A_{M} & A_{T}<A_{M}
\end{array}
$$

Where $A_{T}$ is the True value and $A_{M}$ is the measured value. RAE is zero for optimal segmentation, while it is one if there are zero overlaps of the object areas. The results are calculated by measuring RAE for various segmentation methods viz. Otsu's method, K-Means clustering, and Fuzzy C-Means clustering method. We can conclude that better performance is observed in the quantitative aspect in terms of area and RAE of defects. It is observed that the proposed method has a higher value for no. of defects detected and a lower value for RAE. The Area and RAE values of defects of various lateral sizes and depths are tabulated in Table.1.

\section{RESULTS AND CONCLUSION}

The proposed segmentation algorithm for Adaptive Thresholding is applied to the image extracted from the Thermogram. This is an image of a specimen of Glass Fiber Reinforced Polymer, taken using the technique of FMTWI [14], in which 25 Teflon inserts of different dimensions are integrated at different depths. The results obtained after the application of the proposed thresholding method demonstrate that quantitative measures have shown good performance. The segmented image shows more delamination data when compared to the thermogram. The proposed segmentation assumes that some parts remain under more shadow in an image and thus illuminations affect the image as well. A global threshold value is taken as the mean value in the conventional method of thresholding. In an image, if there is a pixel value greater than the threshold value in a darker region, that portion of the image appears in the foreground. Similarly, if the value is lower than the threshold value, then the background displays that pixel or part. The resulting image of adaptive thresholding is a binary image, as it illustrates the differences between different threshold values. The white region describes values below the threshold, and the black region describes values above the threshold. The Raw image obtained from the Thermal Camera is subjected to Adaptive Segmentation and the fusion technique is used in Thermographic Images to extract delamination's such as defect field, depth, and RAE on images segmented at different time instants. In performing quantitative analysis on the GFRP specimen image, this process has been successful. The results are determined by RAE calculation for various Viz segmentation techniques. The Otsu process, Adaptive Thresholding, Clustering K-means, and FCM. The tabulated results indicate that, when compared to other segmentation methods, the proposed method may distinguish all the defects in the specimen object. The proposed algorithm is capable of recognizing all of the image's 25 defects. It is inferred from the following findings that the method proposed showed better performance in terms of lateral scale, area, and RAE of defects in the quantitative aspect.

Published By: Blue Eyes Intelligence Engineering DOI:10.35940/ijeat.C2241.0210321

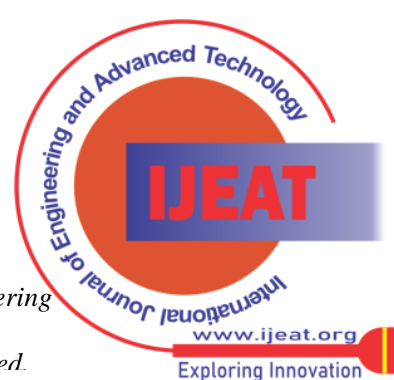




\section{Defect Detection Based on Segmentation of Thermographic Images of Frequency Modulated Thermal Wave Imaging}

Table-1: Comparison of Area and RAE of Each Defect

\begin{tabular}{|c|c|c|c|c|c|c|c|c|c|c|}
\hline \multicolumn{11}{|c|}{ RAE values Defect of lateral size $15 \mathrm{~mm}$ (Area: $2.25 \mathrm{~cm}^{2}$ ) } \\
\hline \multirow{3}{*}{ Method } & \multicolumn{10}{|c|}{ Depth (in mm) } \\
\hline & \multicolumn{2}{|c|}{0.1} & \multicolumn{2}{|c|}{0.3} & \multicolumn{2}{|c|}{0.5} & \multicolumn{2}{|c|}{0.7} & \multicolumn{2}{|c|}{0.9} \\
\hline & Area & RAE & Area & RAE & Area & RAE & Area & RAE & Area & RAE \\
\hline Otsu & 2.27 & 0.01 & 1.12 & 0.50 & 1.63 & 0.27 & 1.76 & 0.21 & ND & 1 \\
\hline Adoptive. thresholding & 1.26 & 0.43 & 1.38 & 0.40 & 1.98 & 0.11 & 1.84 & 0.18 & 0.14 & 0.93 \\
\hline K means & 2.38 & 0.05 & 1.32 & 0.41 & 1.74 & 0.22 & 1.94 & 0.14 & ND & 1 \\
\hline FCM & ND & 1 & 1.76 & 0.21 & 1.96 & 0.12 & 2.30 & 0.25 & 0.07 & 0.97 \\
\hline Proposed & 1.28 & 0.42 & 1.34 & 0.40 & 1.98 & 0.12 & 1.78 & 0.20 & 0.14 & 0.94 \\
\hline
\end{tabular}

RAE values Defect of lateral size $7 \mathrm{~mm}$ (Area: $0.49 \mathrm{~cm}^{2}$ )

\begin{tabular}{|c|c|c|c|c|c|c|c|c|c|c|}
\hline \multirow[t]{3}{*}{ Method } & \multicolumn{10}{|c|}{ Depth (in mm) } \\
\hline & \multicolumn{2}{|l|}{0.1} & \multicolumn{2}{|l|}{0.3} & \multicolumn{2}{|l|}{0.5} & \multicolumn{2}{|l|}{0.7} & \multicolumn{2}{|l|}{0.9} \\
\hline & Area & RAE & Area & RAE & Area & RAE & Area & RAE & Area & RAE \\
\hline Otsu & 1.38 & 0.62 & Q1.29 & 0.41 & 0.67 & $0.5 B$ & 0.98 & 0.82 & ND & 1 \\
\hline Adoptive. thresholding & $0.7 \theta$ & 0.36 & 0.53 & 0.08 & 0.99 & 0.01 & 0.80 & 0.68 & 0.07 & 0.83 \\
\hline K means & 1.82 & $0.3 z$ & $0.3 z$ & 0.82 & 0.88 & 0.38 & 0.02 & 0.04 & ND & 1 \\
\hline FCM & ND & 1 & 0.39 & $0 . \theta \theta$ & 0.92 & 0.38 & 0.08 & 0.69 & ND & 1 \\
\hline Proposed & 0.89 & 0.39 & 0.58 & 0.08 & 0.92 & 0.00 & Q.68 & 0.28 & 0.08 & 0.91 \\
\hline
\end{tabular}

\section{RAE values Defect of lateral size $5 \mathrm{~mm}$ (Area: $0.25 \mathrm{~cm}^{2}$ )}

\begin{tabular}{|c|c|c|c|c|c|c|c|c|c|c|}
\hline \multirow[t]{3}{*}{ Method } & \multicolumn{10}{|c|}{ Depth (in mm) } \\
\hline & \multicolumn{2}{|l|}{0.1} & \multicolumn{2}{|l|}{0.3} & \multicolumn{2}{|l|}{0.5} & \multicolumn{2}{|l|}{0.7} & \multicolumn{2}{|l|}{0.9} \\
\hline & Area & RAE & Area & RAE & Area & RAE & Area & RAE & Area & RAE \\
\hline Otsu & 0.02 & 0.91 & 0.01 & 0.955 & 0.4 & 0.6 & ND & 1 & ND & 1 \\
\hline Adoptive. thresholding & 0.32 & 0.22 & 0.08 & 0.64 & 0.32 & 0.22 & 0.33 & 0.24 & 0.02 & 0.91 \\
\hline K means & 0.02 & 0.091 & 0.09 & 0.64 & 0.45 & 0.45 & ND & 1 & ND & 1 \\
\hline FCM & 0.05 & 0.78 & 0.17 & 0.33 & 0.61 & 0.60 & ND & 1 & ND & 1 \\
\hline Proposed & 0.30 & 0.16 & 0.13 & 0.46 & 0.34 & 0.27 & 0.33 & 0.24 & 0.07 & 0.69 \\
\hline \multicolumn{11}{|c|}{ RAE values Defect of lateral size $3 \mathrm{~mm}$ (Area: $0.09 \mathrm{~cm}^{2}$ ) } \\
\hline \multirow[t]{3}{*}{ Method } & \multicolumn{10}{|c|}{ Depth (in mm) } \\
\hline & \multicolumn{2}{|l|}{0.1} & \multicolumn{2}{|l|}{0.3} & \multicolumn{2}{|l|}{0.5} & \multicolumn{2}{|l|}{0.7} & \multicolumn{2}{|l|}{0.9} \\
\hline & Area & RAE & Area & RAE & Area & RAE & Area & RAE & Area & RAE \\
\hline Otsu & ND & 1 & 0.07 & 0.14 & ND & 1 & ND & 1 & ND & 1 \\
\hline Adoptive. thresholding & 0.19 & 0.52 & 0.01 & 0.87 & ND & 1 & 0.26 & 0.66 & ND & 1 \\
\hline K means & ND & 1 & 0.16 & 0.45 & ND & 1 & ND & 1 & ND & 1 \\
\hline FCM & ND & 1 & 0.36 & 0.75 & ND & 1 & ND & 1 & ND & 1 \\
\hline Proposed & 0.22 & 0.60 & 0.08 & 0.01 & 0.01 & 0.87 & 0.21 & 0.57 & ND & 1 \\
\hline
\end{tabular}

\section{REFERENCES}

1. S. GHOLIZADEH, A Review of Non-Destructive Testing Methods of Composite Materials, Procedia Structural integrity (J), 2016 ELSEVIER, Vol-1, Pages: 50-57.

2. KRISHNENDU CHATERJEE, SUNEET TULI, SIMON G. PICERING, DARRYL P. ALMOND, A Comparison of the Pulsed, Lock-In and Frequency Modulated Thermography Non-Destructive

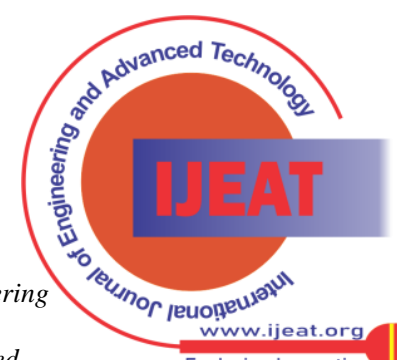


4. KEMAL POLAT, Histogram-based automatic segmentation of images, Neural Computing and Applications July 2016, Volume 27, Issue 5, pp 1445-1450.

5. Ghosha P, Mitchell M, Gold J. Segmentation of thermographic images of hands using a genetic algorithm. In David Fofi KSN. Image Processing: Machine Vision Applications.: SPIE-IS\&T; 2010.

6. Sujji G, Lakshmi YVS, Wiselin G. MRI Brain Image Segmentation based on Thresholding. International Journal of Advanced Computer Research. 2013,3(1):97-101.

7. M.SEZGIN, B. SANKUR, Selection of Thresholding Methods for Non-Destructive Testing Applications, IEEE Proceedings 2001 International Conference on Image Processing, Thessaloniki, Greece.

8. OTSU N, A Threshold selection method from grey level histogram [J], IEEE Trans. Syst 1979, 9(1): 62-66.

9. Kante Murali, Ramakoti Reddy D.V. "Segmentation of Thermographic Sequences in FMTWI for NDE of GFRP", Transactions of Nanjing University of Aeronautics and Astronautics, Vol.35, No.2, April,2018.

10. PRIYANSH SHARMA AND JENKIN SUJI, A Review on Image Segmentation with its Clustering Techniques, International Journal of Signal Processing, Image Processing and Pattern Recognition Vol.9, No.5 (2016), pp.209-218.

11. A. Duartea, L. Carrãob, c, M. Espanhab, T. Vianad, D. Freitasd, P. Bártolod, e, f, P. Fariaa, d, H.A. Almeidaa, d,'Segmentation algorithms for thermal images', CENTERIS 2014 Procedia Technology 16 (2014), Elsevier.

12. Kante Murali, D. V. Rama Koti Reddy And Ravibabu Mulaveesala, Application of image fusion for the IR images in frequency modulated thermal wave imaging for Non-Destructive Testing (NDT) Materials Today: Proceedings 5 (2018) 544-549 ELSEVIER

13. SEZGIN M, SANKUR B, Survey over Image thresholding techniques and quantitative performance evaluation[J]. Journal of Electronic Imaging,2004, 13(I): 146-168.

14. Mulaveesala, R., and Tuli, S., Theory of frequency modulated thermal wave imaging for nondestructive subsurface defect detection (2006) Applied Physics Letters, 89 (19), art. no. 191913.

\section{Authors ProfiLe}

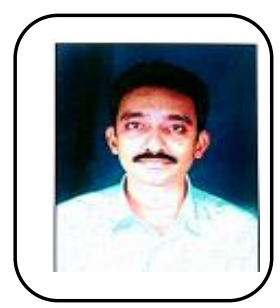

Mr.V. Phani Bhushan had obtained his M. Tech from JNTU College of Eng. Anantapur, India in 2009. He has teaching experience of 19 yrs.in the department of ECE. He is a member of IETE, New Delhi. Currently he is pursuing $\mathrm{PhD}$ in Image processing. His area of Interest includes Digital Electronics, Microprocessors and Field theory etc.

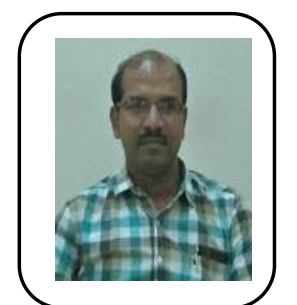

Dr.K. Murali received Ph.D. from Andhra university, Visakhapatnam, India in 2018. He completed M. Tech from IIT, Kanpur in December 2004. Currently he is working as Professor \& Head, Department of ECE, Narayana Engineering College, Nellore, India. He had published more than 25 papers in peer reviewed National \& International journals. His teaching and research interests are Thermal imaging, MEMS and VLSI.

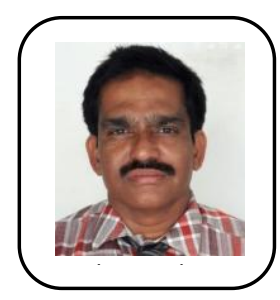

Dr.K.S. SagarReddy did his B. Tech at KLCollege of Engineering and $M$. Tech from JNTU college of Engineering, Anantapur. Completed PhD in Wireless Communications at S.V. University Tirupati in 2016. He has about 15 years of Teaching Experience in the field of Electronics \& Comm.Engg. He published several papers in international Journals. His areas of interest include Wireless Communication.

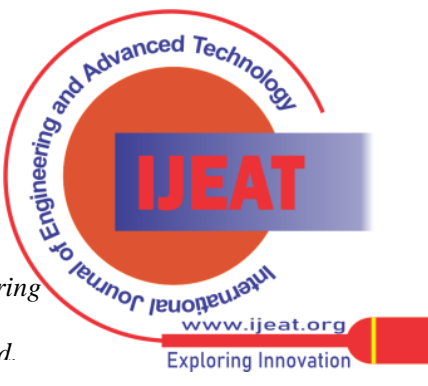

